\title{
Data-based PID controller design for oscillatory systems using PICS method
}

\author{
Yinan Zhang ${ }^{1}$, Thanh Tung Duong Huynh ${ }^{2}$, Min-Sen Chiu ${ }^{2}$ \\ ${ }^{1}$ Department of Chemical \& Biomolecular Engineering, National University of Singapore \\ E5-03-35 4 Engineering Drive 4, Singapore, Singapore \\ e0005490@u.nus.edu; e0115931@u.nus.edu \\ ${ }^{2}$ Department of Chemical \& Biomolecular Engineering, National University of Singapore \\ E5-03-35 4 Engineering Drive 4, Singapore, Singapore \\ checms@nus.edu.sg
}

\section{Extended Abstract}

Although PID controllers are widely implemented in the process industries, only a few PID tuning methods specifically developed to cope with the oscillatory output response inherent in under-damped dynamic systems. In the literature, several PID design methods are developed for the control of underdamped processes. One widely used input shaping technique is Posicast Input Command Shaping (PICS) proposed by Smith [1]. The mechanism of PICS is to add a PICS term before the process to attenuate oscillatory output. By combining the PICS and Magnitude Optimum Multiple Integration (MOMI) methods, Vrancic and Oliveira [2] developed a PID tuning method to remove oscillatory response in openloop dynamics and subsequently calculate the PID tuning parameters. However, the design procedure for this method is also sophisticated and the resulting control performance is not always desirable in terms of overshoot. Moreover, PID tuning methods capable of handling under-damped processes were also developed under Internal Model Control (IMC) framework [3-5]. However, the control performance for those methods will degrade for high-order under-damped processes with heavy oscillations.

On the other hand, the aforementioned tuning methods for under-damped processes are all model-based controller design methods, which all require an accurate process model. In contrast, data-based PID controller design methods can design controllers without the process identification. Campi et al. [6] proposed the virtual reference feedback tuning (VRFT) method in the discrete time domain to design controllers without a process model. The attractive features of the VRFT method are the direct design of controller based on input and output data without iterations. Additionally, Yang et al. [7] extended VRFTbased PID controller design method to the continuous-time systems using a reference model containing time delay as a single tuning parameter. However, when the processes have considerable oscillatory output response, this method will give unsatisfactory control performance. This motivates us to extend VRFT method in conjunction with PICS to achieve better control performance for under-damped processes.

In this study, we have successfully developed a systematic data-based method for under-damped processes. In order to evaluate the performance of the proposed method, the IMC-based PID controller design methods developed by Chien [4], and Lee et al. [5], and Vrancic and Oliveira's MOMI-PICS tuning method [2] are selected as benchmarks to conduct a comparative study for some under-damped cases. The obtained results reveal that the proposed method can achieve more satisfactory control performance compared with its counterparts. Therefore, the proposed method is able to design appropriate controllers for under-damped processes based on the process data.

\section{References}

[1] O. J. Smith, "Posicast control of damped oscillatory systems," Proceedings of the IRE, vol. 45, no. 9, pp. 1249-1255, 1957. 
[2] D. Vrančić and P. M. Oliveira, "Design of feedback control for underdamped systems," IFAC Proceedings Volumes, vol. 45, no. 3, pp. 98-103, 2012.

[3] D. E. Rivera, M. Morari, and S. Skogestad, "Internal model control: PID controller design," Industrial \& engineering chemistry process design and development, vol. 25, no. 1, pp. 252-265, 1986.

[4] I.-L. Chien, "Consider IMC tuning to improve controller performance," Chem. Eng. Prog., vol. 86, pp. 33-41, 1990.

[5] Y. Lee, S. Park, M. Lee, and C. Brosilow, "PID controller tuning for desired closed-loop responses for SI/SO systems," Aiche journal, vol. 44, no. 1, pp. 106-115, 1998.

[6] M. C. Campi, A. Lecchini, and S. M. Savaresi, "Virtual reference feedback tuning: a direct method for the design of feedback controllers," Automatica, vol. 38, no. 8, pp. 1337-1346, 2002.

[7] X. Yang, B. Xu, and M.-S. Chiu, "PID controller design directly from plant data," Industrial \& Engineering Chemistry Research, vol. 50, no. 3, pp. 1352-1359, 2010. 